\title{
On RFID Tag Detection Inside Metal Pipes
}

\author{
Andrea Michel, Paolo Nepa \\ Dept. Information Engineering \\ University of Pisa \\ Pisa, Italy \\ andrea.michel[paolo.nepa]@iet.unipi.it
}

\author{
Marcos Rodriguez Pino \\ Dept. Ingenieria Electrica \\ Universidad de Oviedo \\ Gijon, Spain \\ mpino@uniovi.es
}

\begin{abstract}
In this paper, the detection of a passive UHF RFID tag is analyzed when the tag is located inside a metallic cylindrical pipe. By modeling the pipe with a cylindrical waveguide, it is possible to relate the tag detection with the propagation of a TE or TM mode inside the guided structure. Simulated and measured results are here described.
\end{abstract}

Keywords-UHF RFID, metal pipe, RFID propagation model

\section{INTRODUCTION}

Radio Frequency Identification (RFID) systems have received a large interest in recent years [1], in particular for logistic, retail and pharmaceutical applications. In this context, two different frequency bands are typically used: High Frequency (HF, $13.56 \mathrm{MHz}$ ) and Ultra High Frequency (UHF, $865-928 \mathrm{MHz}$ ) RFID bands. HF RFID systems are employed for short-range applications (e.g. proximity identification and payments) and they are usually based on inductive coupling between coils. On the other hand, UHF RFID systems are used for both short-range [2], [3] and long-range applications, and largely used for identification, localization [4], sensing and Item Level Tagging (ILT) applications. Efforts have been made to create a reliable theoretical model to predict the tag detection by means of numerical results, so improving the overall system performance [3]. However, while the coupling model for longrange UHF RFID is typically based on ray propagation models [1], more accurate and specific models are required for other scenarios, such as near-field applications [3].

UHF RFID systems may be used to identify metal pipes for logistic applications (Fig. 1). On-metal tags can be attached to the external part of the pipe, so that they can be easily detected by means of a portable reader. However, the presence of adjacent pipes may affect the tag readability. Also, atmospheric agents may further deteriorate the tag mechanical conditions. Consequently, the tag is often placed inside the pipe. In this context, metallic pipes can be approximated by a guided structure (e.g. cylindrical waveguide), and the analysis of the mode propagation inside the waveguide becomes crucial for the tag detection.

In this paper, theoretical considerations are discussed to model the detection of a tag placed inside a metal pipe approximated by a guided structure. Also, numerical results and measurements are shown by considering a commercial dipolelike tag. The detection of RFID tags inside guided structures has been already addressed in the past [5]. The aim of this paper is to discuss preliminary considerations suitable to create a more accurate propagation model of UHF RFID signal in guided structures.

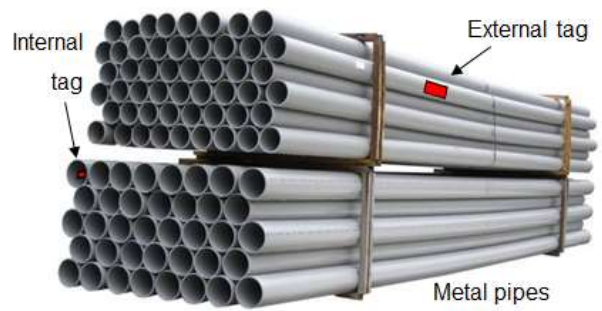

Fig. 1. Example of a set of metal pipes equipped with internal or external onmetal UHF RFID tags.

\section{NumericAl Model AND PRELIMINARY RESUltS}

A metallic pipe can be approximated by a cylindrical structure with radius $a$ and length $L$, as shown in Fig. 2. The UHF RFID tag can be placed inside the pipe, at a distance $R$ from the cylinder axis (i.e. at a distance $D=a-R$ from the external conductor). By using a thick insulator substrate, an inlay UHF RFID tag can be directly attached to the internal surface of the metallic conductor.

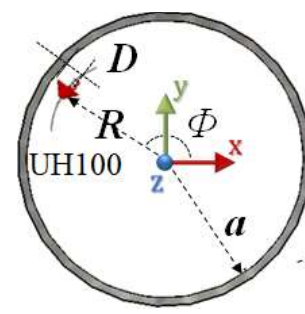

(a)

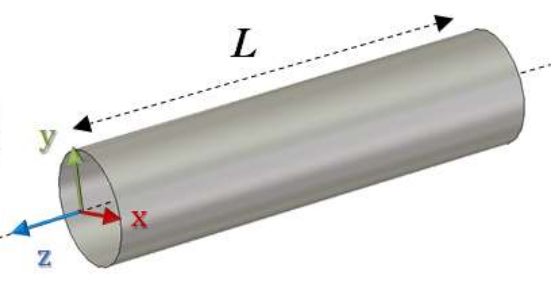

(b)
Fig. 2. Pipe numerical model: (a) transverse view and (b) 3D view.

The value of the cylinder radius, $a$, is a key parameter for the problem analysis. Specifically, it is strictly related to the number of propagating modes inside the guided structure. $\mathrm{TE}_{11}$ is the fundamental mode in a cylindrical waveguide (Fig. 3), and at ETSI UHF RFID band (865-868 MHz) it can propagate if the radius $a$ is higher than $101 \mathrm{~mm}$. It is worth noting that for dipolelike tags, the distribution of the incident electric field is important to predict the RFID chip activation and tag detection [3]. By considering pipes with $101<a<132 \mathrm{~mm}$, only the fundamental mode $\mathrm{TE}_{11}$ can propagate, whose electric field distribution is shown in Fig. 3. In order to consider another TE mode propagating inside the waveguide it is necessary to consider pipes with $a>168 \mathrm{~mm}$. This gives an important 
information for a practical use of RFID systems in metal pipes. In general, three tag positions and orientation can be considered inside a pipe: radial, transversal and longitudinal (Fig. 4). In order to have other TE modes propagating inside the waveguide it is necessary to consider pipes with $a>168 \mathrm{~mm}$.

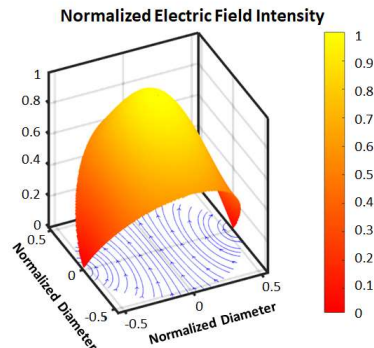

(a)

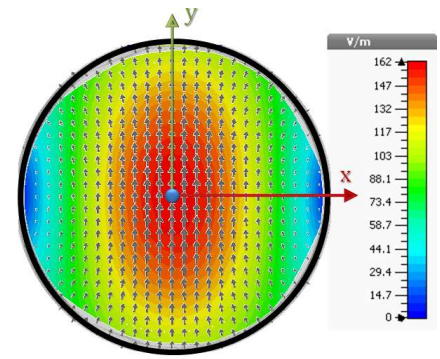

(b)
Fig. 3. Electric field distribution of the fundamental $\mathrm{TE}_{11}$ mode: (a) $3 \mathrm{D}$ and (b) $2 \mathrm{D}$ view.

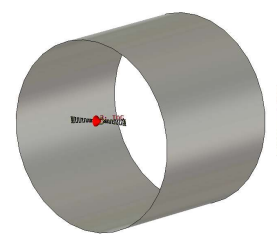

(a)

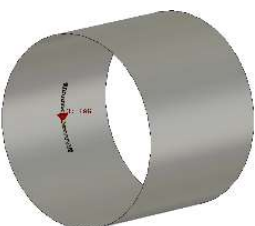

(b)

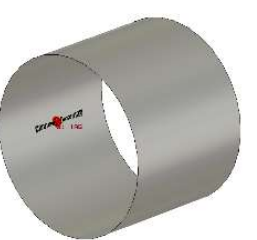

(c)
Fig. 4. Three tag positions and orientation can be considered inside a pipe: (a) radial, (b) transversal and (c) longitudinal.

In Fig. 5, the simulated coupling $\left(\mathrm{S}_{12}\right.$ parameter) between an UH100 Inlay (LabID) tag model and the fundamental mode (TE $E_{11}$ ) propagating in a cylindrical waveguide with $a=12.5 \mathrm{~mm}$ is shown as a function of the $\phi$ angle (Fig. 2a), at a frequency of $866 \mathrm{MHz}$. The numerical results have been obtained by using CST Microwave Studio.

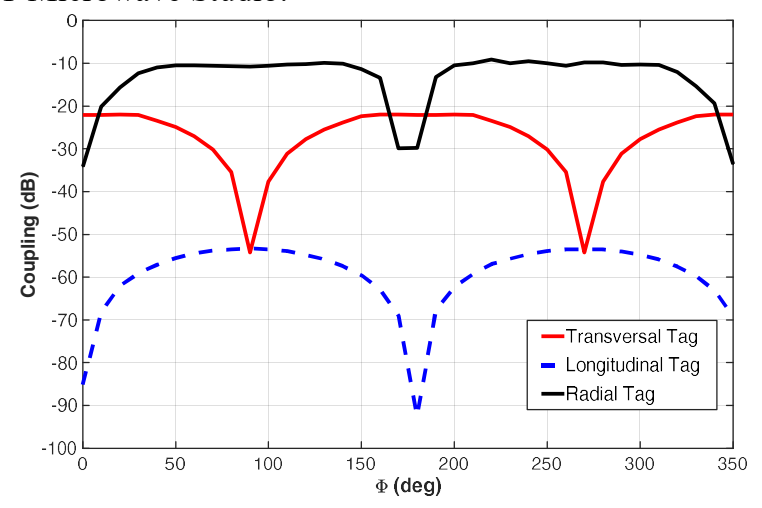

Fig. 5. Simulated coupling ( $\mathrm{S}_{12}$ parameter) between an UH100 Inlay (LabID) tag model located at $D=25 \mathrm{~mm}$ and the fundamental mode $\left(\mathrm{TE}_{11}\right)$ propagating in a cylindrical waveguide with $a=12.5 \mathrm{~mm}$, as a function of the $\phi$ angle.

It is evident that the coupling between the fundamental mode and a dipole-like tag placed in a longitudinal position (Fig. 4c) is definitely low, since the longitudinal component of the electric field vanishes for the TE modes. The higher coupling with a dipole-like tag can be achieved by placing the tag in a radial position (Fig. 4b), if properly aligned with the mode polarization (Fig. 3b). However, for this tag position, mechanical issues may occur, since a proper structure should be created inside the pipe - the tag cannot be directly attached to the cylindrical structure. A fair compromise is represented by the transversal position (Fig. 4a), by including an insulator substrates between the inlay flexible tag and the metallic structure. In this framework, in Fig. 6 the measured tag-mode coupling is represented as a function of the distance $D$ between the internal surface of the cylindrical pipe $(a=12.5, L=2 \mathrm{~m})$ and the UH100 tag in a transversal position (Fig. 4b). A foam substrate is used to attach the tag to the metallic cylinder. The tag-mode coupling is derived by considering the reader output power, the reader antenna gain, the RFID chip sensitivity and the attenuation introduced by the metallic pipe.

Above considerations can be taken into account to create a theoretical tag detection model when UHF RFID tags are placed inside cylindrical structures that can be modeled with rectangular/circular waveguides or coaxial cables.

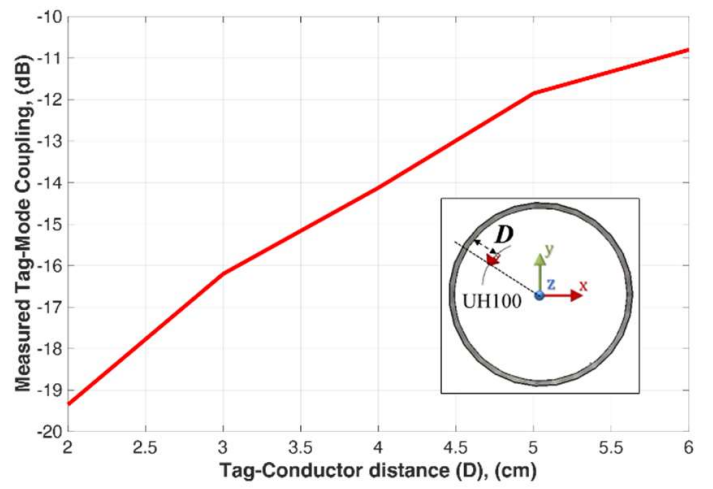

Fig. 6. Measured tag-mode coupling as a function of the distance $D$ between a transversal UH100 tag and the internal surface of the metallic cylindrical pipe.

\section{ACKNOWLEDGEMENTS}

This work was supported in part by the Ministerio de Educación, Cultura y Deporte - Programa de Movilidad Salvador de Madariaga (Ref. PRX17/00635); by the Ministerio de Economía y Competitividad, under projects TEC2014-54005-P (MIRIIEM) and TEC2017-86619-R (ARTEINE) and by the Gobierno del Principado de Asturias/FEDER under project GRUPIN14-114.

\section{REFERENCES}

[1] K. Finkenzeller, "RFID Handbook: Fundamentals and Applications in Contactless Smart Cards and Identification", 3rd ed. Hoboken, NJ: Wiley, 2010.

[2] A. Michel, P. Nepa, X. Qing and Z.-N. Chen, "Considering HighPerformance Near-Field Reader Antennas: Comparisons of Proposed Antenna Layouts for Ultrahigh-Frequency Near-Field Radio-Frequency Identification", to appear on IEEE Antennas and Propagation Magazine, 2018.

[3] A. Michel, M. Rodriguez-Pino, and P. Nepa, "Reconfigurable modular antenna for near-field UHF RFID smart point readers," IEEE Trans. Antennas Propag., vol. 65, no. 2, pp. 1-9, Feb. 2017.

[4] A. Buffi, M. R. Pino and P. Nepa, "Experimental Validation of a SARBased RFID Localization Technique Exploiting an Automated Handling System," IEEE Antennas and Wireless Propagation Letters, vol. 16, pp. 2795-2798, 2017.

[5] A.S. Andrenko. "EM modeling of Metal Pipe Based UHF RFID Tags Activated by R/W Antenna", 2012 International Conference on Electromagnetics in Advanced Applications - ICEEA, Cape Town, South Africa, 2012. 